\title{
$W$ plus two jets from a quasi-inert Higgs doublet
}

\author{
Qing-Hong Cao, ${ }^{a, b}$ Marcela Carena, ${ }^{b, c}$ Stefania Gori, ${ }^{b}$ Arjun Menon, ${ }^{d}$ \\ Pedro Schwaller, ${ }^{a, e}$ Carlos E.M. Wagner ${ }^{a, b, f}$ and Lian-Tao Wang ${ }^{b, f}$ \\ ${ }^{a}$ HEP Division, Argonne National Laboratory, \\ 9700 Cass Ave., Argonne, IL 60439, U.S.A. \\ ${ }^{b}$ Enrico Fermi Institute, University of Chicago, \\ Chicago, IL 60637, U.S.A. \\ ${ }^{c}$ Theoretical Physics Department, Fermilab, \\ Batavia, IL 60510, U.S.A. \\ ${ }^{d}$ Illinois Institute of Technology, \\ Chicago, IL 60616, U.S.A. \\ e Physics Department, University of Illinois at Chicago, \\ Chicago, IL 60607, U.S.A. \\ ${ }^{f}$ Kavli Institute for Cosmological Physics, University of Chicago, \\ Chicago, IL 6063\%, U.S.A. \\ E-mail: qinghongcao@gmail.com, carena@fnal.gov, goris@uchicago.edu, \\ aamenon@agni.phys.iit.edu, pschwaller@hep.anl.gov, \\ cwagner@hep.anl.gov, liantaow@uchicago.edu
}

ABSTRACT: We show that, the result recently reported by the CDF collaboration showing an excess in the invariant mass distribution of jet pairs produced in association with a $W$-boson can be explained by a simple extension of the Standard Model (SM) with an additional quasi-inert Higgs doublet. The two additional neutral Higgs states $H^{0}$ and $A^{0}$ have a mass of about $150 \mathrm{GeV}$ and decay into a pair of jets. $W^{ \pm} H^{0} / A^{0}$ pairs are produced from the decay of the heavier charged Higgs boson $H^{ \pm}$. Depending on the precise masses of the neutral and charged Higgs bosons, the model is shown to be in agreement with constraints from electroweak precision tests and from flavor physics for a broad range of the Standard Model-like Higgs mass from $100 \mathrm{GeV}$ to several hundreds of GeV. Other possible signals of this model at the Tevatron and the LHC are discussed.

Keywords: Beyond Standard Model, Higgs Physics

ARXIV EPRINT: 1104.4776 


\section{Contents}

1 Introduction 1

2 The model 2

3 Electroweak precision constraints and flavor $\quad 4$

$4 W^{+} H^{0} / W^{+} A^{0}$ production and $\mathrm{W}+$ jets excess $\quad 6$

5 Prospects at Tevatron and LHC 9

$\begin{array}{llr}6 & \text { Conclusions } & 10\end{array}$

$\begin{array}{ll}\text { A Alternative spectrum } & 11\end{array}$

\section{Introduction}

Recently the CDF collaboration has reported an excess in the dijet invariant mass distribution in events where a $W$-boson is produced together with two jets. The $W$-boson in this case is required to decay leptonically into electrons or muons. Besides the peak around $M_{j j}=80 \mathrm{GeV}$ from di-boson production with one hadronically decaying $W$-boson, the invariant mass distribution shows an excess in the region $120 \mathrm{GeV}<M_{j j}<160 \mathrm{GeV}$. This disagrees with the Standard Model (SM) background at the $3.2 \sigma$ level and is consistent with a dijet resonance with a mass of $144 \pm 5 \mathrm{GeV}[1]$.

The CDF excess can readily be explained by introducing an additional particle that couples to the first generation of quarks, e.g. using a $Z^{\prime}$ boson [2-9]. In this case one has to worry about a potentially large contribution also in the $Z+2$ jets and $\gamma+2$ jets channels, which are only suppressed by electroweak mixing angles and by the branching fraction of the $Z$ boson into leptons.

Another promising approach is to introduce a second resonance that is produced onshell and decays into a $W$-boson and into the dijet resonance. For recent work that implements this and other ideas, see [10-17]. It is also possible that the excess is due to mismodeling of some of the Standard Model backgrounds, e.g. from top production $[18,19]$.

In this paper, we present a simple and renormalizable model that implements the two resonance approach in a compact way. In addition to the Standard Model particle content, just one complex electroweak scalar doublet is introduced. In the next section, we introduce the basic features of the model. In section 3 we discuss constraints from electroweak precision tests and from flavor physics. In section 4 we show that the model can explain the excess reported by $\mathrm{CDF}$, and, in section 5 , we discuss possible other signals at the Tevatron and at the LHC. We reserve section 6 for our conclusions and we present an 
appendix, in which we analyze the possible consequences of a different mass arrangement for the charged and neutral Higgs bosons of the theory.

\section{The model}

We consider a simple extension of the Standard Model by including an additional scalar doublet $\Phi_{2}$, with the same hypercharge as the SM doublet $\Phi_{1}$. Consider the renormalizable scalar potential of $\Phi_{1}=\left(\phi^{-}, \phi^{0}\right)$ and $\Phi_{2}=\left[H^{-},\left(H^{0}+i A^{0}\right) / \sqrt{2}\right]$,

$$
\begin{aligned}
V= & \mu_{1}^{2} \Phi_{1}^{\dagger} \Phi_{1}+\mu_{2}^{2} \Phi_{2}^{\dagger} \Phi_{2}+\left(\mu_{12}^{2} \Phi_{1}^{\dagger} \Phi_{2}+\text { h.c. }\right)+\frac{1}{2} \lambda_{1}\left(\Phi_{1}^{\dagger} \Phi_{1}\right)^{2}+\frac{1}{2} \lambda_{2}\left(\Phi_{2}^{\dagger} \Phi_{2}\right)^{2} \\
& +\lambda_{3}\left(\Phi_{1}^{\dagger} \Phi_{1}\right)\left(\Phi_{2}^{\dagger} \Phi_{2}\right)+\lambda_{4}\left(\Phi_{1}^{\dagger} \Phi_{2}\right)\left(\Phi_{2}^{\dagger} \Phi_{1}\right)+\frac{1}{2} \lambda_{5}\left(\Phi_{1}^{\dagger} \Phi_{2}\right)^{2}+\frac{1}{2} \lambda_{5}^{*}\left(\Phi_{2}^{\dagger} \Phi_{1}\right)^{2},
\end{aligned}
$$

where we have assumed that all quartic couplings involving an odd number of $\Phi_{1}$ or $\Phi_{2}$ fields are suppressed (see discussion below). All the above parameters are necessarily real except $\lambda_{5}$ and $\mu_{12}^{2}$. We note also that vacuum stability imposes the requirements

$$
\lambda_{1,2}>0, \quad \lambda_{3}>-\sqrt{\lambda_{1} \lambda_{2}}, \quad \lambda_{3}+\lambda_{4}-\left|\lambda_{5}\right|>-\sqrt{\lambda_{1} \lambda_{2}} .
$$

These conditions are easily fulfilled for the range of parameters relevant to this work.

We assume that $\Phi_{1}$ and $\Phi_{2}$ interact with the SM quarks via the Yukawa interaction

$$
\mathcal{L} \supset y_{u, 1} \Phi_{1} Q u+y_{d, 1} \Phi_{1}^{c} Q d+y_{u, 2} \Phi_{2} Q u+y_{d, 2} \Phi_{2}^{c} Q d,
$$

where $Q=\left(u_{L}, d_{L}\right)^{c}$ denotes the SM quark doublet while $u$ and $d$ are the SM quark singlets. To avoid experimental constraints from the lepton sector, at this point we choose not to couple $\Phi_{2}$ to leptons.

We demand that $\Phi_{2}$ develops a vanishing (or negligible) vacuum expectation value (VEV). In other words $\Phi_{2}$ is not involved in the electroweak symmetry breaking. The SM $\mathrm{SU}(2) \times \mathrm{U}(1)_{Y}$ gauge symmetry is spontaneously broken by $\left\langle\phi^{0}\right\rangle=v=174 \mathrm{GeV}$. In order to forbid a VEV for $\Phi_{2}$ at tree-level it suffices to set the $\mu_{12}$ term in the scalar potential to zero. At one loop however, the $\mu_{12}$ term will be generated by radiative corrections:

$$
\delta \mu_{12}^{2}=\frac{y_{u, 1}^{\dagger} y_{u, 2}+y_{d, 1}^{\dagger} y_{d, 2}}{16 \pi^{2}} \Lambda^{2}
$$

where $\Lambda$ is a cutoff scale that is naturally expected to be around a few TeV. Assuming that the $\Phi_{2}$ couplings are flavor independent, and of order 0.1 , we get a mixing contribution of the order of $1000 \mathrm{GeV}^{2}$. This mixing term would induce a vacuum expectation value $\left\langle\Phi_{2}\right\rangle$ of order $10 \mathrm{GeV}$, leading to too large contributions of $\Phi_{2}$ to the light quark masses. In order to avoid such a large contribution, a mixing mass $\mu_{12}^{2}$ smaller than about $10 \mathrm{GeV}^{2}$ would be required, what may be obtained in the case of flavor independent couplings by a cancellation between the tree-level and loop-induced contributions, requiring a large fine tuning. It is however possible that $\Phi_{2}$ only couples to the first generation quarks. In such case, provided that $\mu_{12}^{2}$ is set to zero at the tree-level by some symmetry, no large fine tuning would be required. 
To explicitly forbid the mixing between the two Higgs doublets, we can impose a $\mathbf{Z}_{2}$ parity symmetry under which $\Phi_{2}$ is odd [20,21], leading also to a justification of the suppression of the quartic couplings with odd powers of the fields $\Phi_{1,2}$ in the scalar potential (eq. (2.1)). This is similar to what is obtained in R-Parity violating models, with $\Phi_{2}$ replaced by a slepton [11]. However, R-Parity violating models of this type are strongly constrained by the possible generation of neutrino masses (for a recent analysis, see ref. [22]), a constraint that is not present in the two Higgs doublet model case.

To allow a coupling of $\Phi_{2}$ to quarks, we further require that the right-handed up quark is odd under this new parity symmetry, while all the other SM fields are even. A consequence of this is that $\Phi_{1}$ would not couple to the right-handed up quark and therefore the up quark would remain massless at tree-level. Were this parity symmetry preserved, it would lead to a potential solution of the strong CP-problem, which seems to be disfavored by results from chiral perturbation and lattice gauge theory [23-25] (see, however, ref. [26]).

In order to generate a value of the up quark mass of about a few $\mathrm{MeV}$, a soft breakdown of the $Z_{2}$-symmetry, via a non-vanishing $\mu_{12}^{2}$ term of about $10 \mathrm{GeV}^{2}$, would be necessary.

Assuming this approximate $Z_{2}$-symmetry in the Yukawa Lagrangian of eq. (2.3), we can write down the Yukawa couplings that we use for our analysis as

$$
\mathcal{L} \mathcal{Y}=y_{u, 1}^{i \alpha} \Phi_{1} Q_{i} u_{\alpha}+y_{d, 1}^{i j} \Phi_{1}^{c} Q_{i} d_{j}+y_{u, 2}^{i 1} \Phi_{2} Q_{i} u_{1}
$$

Note that the index $\alpha$ can only take the values $\alpha \in\{2,3\}$ since a coupling of the righthanded up quark to $\Phi_{1}$ is forbidden by the parity symmetry. Contrary to what happens in ordinary two Higgs doublet models (2HDMs), these couplings are not proportional to the ratio of quark masses to the SM Higgs vacuum expectation value, in particular $y_{u, 2}^{11}$ can be much larger than in usual $2 \mathrm{HDM}$.

We conclude this section with the Higgs spectrum of the theory:

$$
\begin{aligned}
M_{h}^{2} & =2 \lambda_{1} v^{2}, \\
M_{H^{ \pm}}^{2} & =\mu_{2}^{2}+\lambda_{3} v^{2}, \\
M_{H^{0}}^{2} & =\mu_{2}^{2}+\left(\lambda_{3}+\lambda_{4}+\lambda_{5}\right) v^{2}, \\
M_{A^{0}}^{2} & =\mu_{2}^{2}+\left(\lambda_{3}+\lambda_{4}-\lambda_{5}\right) v^{2} .
\end{aligned}
$$

The lone Higgs boson of the Standard Model is of course $h$, whereas $H^{ \pm}, H^{0}$, and $A^{0}$ are the components of the new scalar doublet.

Using these expressions for the masses of the scalar fields, we can rewrite the condition on the vacuum stability (eq. (2.2)) as a condition on the $\mu_{2}$ parameter

$$
\mu_{2}^{2}<\operatorname{Min}\left(M_{H^{0}}^{2}, M_{A^{0}}^{2}\right)+\sqrt{\frac{\lambda_{2}}{2}} M_{h} v .
$$

Finally, the $Z$-pole precision measurement sets the limit

$$
M_{Z}<M_{H^{0}}+M_{A^{0}} .
$$

There are also bounds coming from the direct searches for the neutral Higgs particles at LEP2, which may increase the above limit in eq. (2.11) from $M_{Z}$ to values close to $200 \mathrm{GeV}$. 


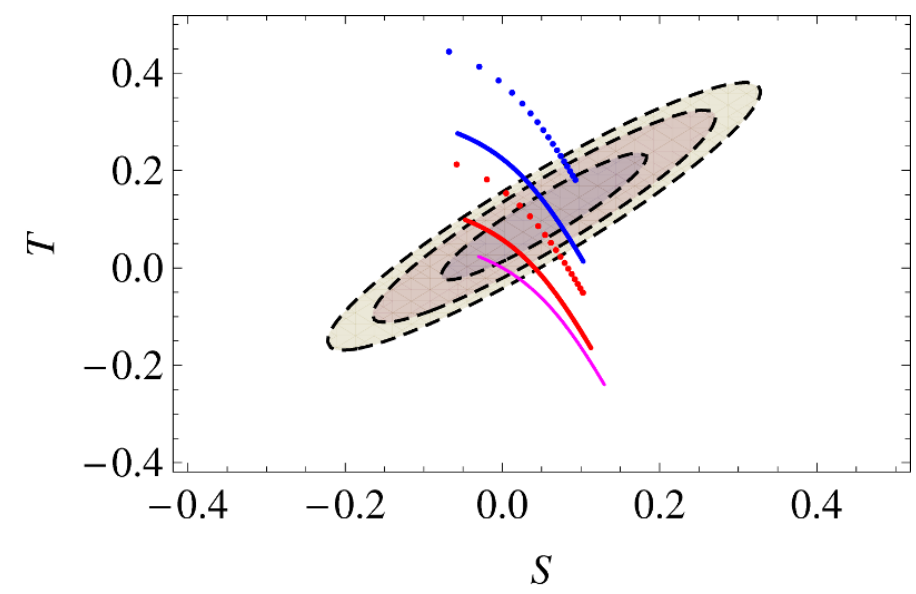

Figure 1. The dashed ellipses show the $68 \%, 95 \%$ and $99 \% \mathrm{CL}$ contours on the (S, T) plane obtained by Gfitter [27]. We fix $M_{H^{0}}=150 \mathrm{GeV}, M_{A^{0}}=210 \mathrm{GeV}$ and the charged Higgs mass to $M_{H^{ \pm}}=300 \mathrm{GeV}$ (blue curve, second from top), $M_{H^{ \pm}}=250 \mathrm{GeV}$ (red curve, fourth from top). Each line shows, from top to bottom, the shift in $(\mathrm{S}, \mathrm{T})$ resulting from increasing the SM-like Higgs mass from $80 \mathrm{GeV}$ to $900 \mathrm{GeV}$. The case of non splitting between $M_{A^{0}}$ and $M_{H^{0}}$ is also shown $\left(M_{H^{0}}=M_{A^{0}}=150 \mathrm{GeV}\right)$ : the blue dotted curve (first from top) is for $M_{H^{ \pm}}=300 \mathrm{GeV}$, the red dotted curve (third from top) for $M_{H^{ \pm}}=250 \mathrm{GeV}$. The magenta curve (last from top) corresponds to the SM.

However, the precise bound on the sum of the neutral Higgs boson masses depends strongly on the decay properties of these particles. In this article, we will be interested in masses for these particles that strongly exceed the precision measurement and LEP2 bounds.

\section{Electroweak precision constraints and flavor}

We perform the analysis of the ElectroWeak Precision Tests (EWPTs) in the S-T plane, with the experimental contours taken from [27]. Since, as we will show in the next section, we have to require a rather large mass splitting between the scalars $H^{0}, A^{0}$ and the charged Higgs $H^{ \pm}$to fit the $W+2$ jets CDF excess, we expect a large positive New Physics (NP) contribution to the $\mathrm{T}$ parameter. To calculate the NP effects to $\mathrm{S}$ and $\mathrm{T}$, we use the general formula valid for $2 \mathrm{HDMs}[28,29]$, under the assumptions of no mixing between the two Higgs doublets and of negligible VEV of the second doublet $\Phi_{2}$. In order to generate a $W+2 j$ signature consistent with observation, we shall fix the mass of one of the scalars to be close to $150 \mathrm{GeV}$.

In figure 1 we present the values of the $S$ and $T$ parameters for varying values of the SM-like Higgs mass $M_{h}$, from $80 \mathrm{GeV}$ to $900 \mathrm{GeV}$, and different values of the $\Phi_{2}$ charged and neutral Higgs bosons masses. Dashed ellipses represent the values of the $\mathrm{S}$ and $\mathrm{T}$ parameters consistent with electroweak precision tests at 68,95 and $99 \%$ confidence level. Fixing a splitting of $150 \mathrm{GeV}$ between the charged Higgs and the neutral Higgs bosons $\left(M_{H^{0}}=\right.$ $150 \mathrm{GeV}, M_{H^{ \pm}}=300 \mathrm{GeV}$ ) and assuming degeneracy between scalar and pseudoscalar masses, a very large mass for the SM Higgs boson $h, M_{h}>650 \mathrm{GeV}$, would be required to obtain consistency with electroweak precision tests at the $2 \sigma$ level (see the blue dotted 

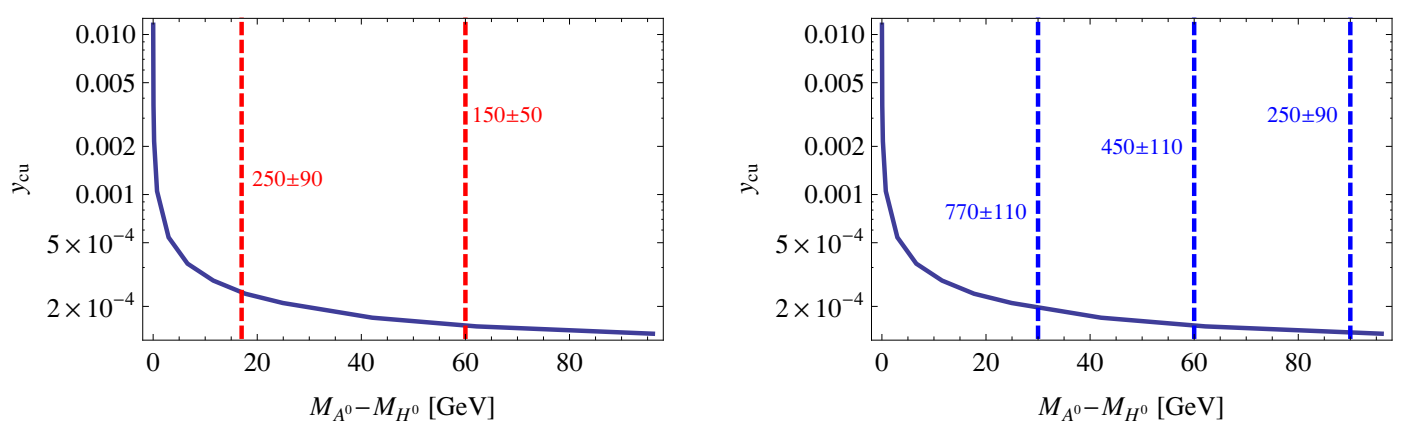

Figure 2. Constraint on the coupling $y_{c u}$ as a function of the mass splitting $M_{A^{0}}-M_{H^{0}}$. The region above the solid line is disfavored at $2 \sigma$ by the present experimental bounds on $D-\bar{D}$ mixing. On the left panel the charged mass is fixed to $250 \mathrm{GeV}$, on the right $M_{H^{ \pm}}=300 \mathrm{GeV}$. The vertical lines indicate the value of the SM Higgs mass $M_{h}$ (in $\mathrm{GeV}$ ) needed in order to satisfy the constraints coming from EWPTs at the $1 \sigma$ level.

curve in figure 1). A good fit, for moderate values of the SM Higgs boson mass $M_{h}$ may be obtained by lowering the mass of the charged Higgs: for $M_{H^{ \pm}}=250 \mathrm{GeV}$ and $M_{H^{0}}=M_{A^{0}}=150 \mathrm{GeV}$, a SM Higgs with mass in the range $(200-480) \mathrm{GeV}$ (and with central value $300 \mathrm{GeV}$ ) would be required to be in agreement with EWPTs at the $2 \sigma$ level (see the red dotted curve in figure 1).

An alternative way of reducing the required SM-like Higgs boson mass is to assume a splitting between the $A^{0}$ and $H^{0}$ masses through a non-vanishing value of $\lambda_{5} .{ }^{1}$ In figure 1 , we show our results once we fix $M_{H^{0}}=150 \mathrm{GeV}, M_{A^{0}}=210 \mathrm{GeV}$ and the charged mass to $M_{H^{ \pm}}=300 \mathrm{GeV}$ (solid blue curve) or $M_{H^{ \pm}}=250 \mathrm{GeV}$ (solid red curve). As we can observe from the figure, a more moderate value of the Higgs mass $M_{h}$ is required in order to be in accordance with EWPTs: the best fit is obtained for $M_{h}=450 \mathrm{GeV}$ (with $2 \sigma$ boundary given by $(290-660) \mathrm{GeV}$ ) in the case of a $300 \mathrm{GeV}$ charged Higgs boson, and for $M_{h}=150 \mathrm{GeV}$ (with $2 \sigma$ boundary given by $(90-250) \mathrm{GeV}$ ) in the case of $M_{H^{ \pm}}=250 \mathrm{GeV}$. Observe that a heavy SM-like Higgs boson with mass up to $400 \mathrm{GeV}$ will be tested at the LHC through its decay into massive gauge bosons within the next two years [30].

Furthermore flavor adds additional constraints on this model. In the mass eigenbasis, the quark- $\Phi_{2}$ Higgs interaction Lagrangian involving the right-handed up quark is

$$
\mathcal{L}^{u_{1}}=y_{u, 2}^{i 1} U_{u, L}^{i j}\left(\phi_{2}^{0} \bar{u}_{L, j} u_{1}-\phi_{2}^{-} V_{\mathrm{CKM}}^{j k} \bar{d}_{L, k} u_{1}\right)
$$

Clearly the GIM mechanism is violated strongly and an assumption about the flavor structure of the Yukawa matrix is needed to avoid large NP effects in flavor violating observables. We choose to make the simplifying assumption, $y_{u, 2}^{i 1}=y_{u, 2} \delta^{i 1}$. We shall analyze two possible limits for the rotation matrix, $U_{u, L}^{i j}=\delta^{i j}$ and $U_{u, L}^{i j}=\left(V_{\mathrm{CKM}}^{i j}\right)^{\dagger}$. Under these assumptions

\footnotetext{
${ }^{1}$ Note however that the constraints coming from flavor physics are in general harder to satisfy in this case, as will be discussed in the following.
} 
the flavor violating Higgs mediated couplings are

$$
\mathcal{L}^{u_{1}}= \begin{cases}y_{u, 2}\left(\phi_{2}^{0} \bar{u}_{L, 1} u_{1}-\phi_{2}^{-} V_{\mathrm{CKM}}^{1 k} \bar{d}_{L, k} u_{1}\right) & \text { for } U_{u, L}^{i j}=\delta^{i j} \\ y_{u, 2}\left(V_{\mathrm{CKM}}^{* j 1} \phi_{2}^{0} \bar{u}_{L, j} u_{1}-\phi_{2}^{-} \bar{d}_{L, 1} u_{1}\right) & \text { for } U_{u, L}^{i j}=V_{\mathrm{CKM}}^{* j i}\end{cases}
$$

For the case $U_{u, L}^{i j}=\delta^{i j}$, the charged Higgs $\phi_{2}^{-}$interactions lead to a new source of flavor violation, beyond the SM one. These flavor violating interactions are governed by the couplings $y_{u, 2} V_{\mathrm{CKM}}^{1 k}$, and depending on the $y_{u, 2}$ value, tree-level flavor violating processes like $K^{+} \rightarrow \pi^{+} \pi^{0}$ can put strong constraints on this scenario. The contribution to the $K^{+} \rightarrow \pi^{+} \pi^{0}$ mode due to the charged Higgs, as compared to the Standard Model, is naively suppressed by the factor $\left(y_{u, 2} / g_{2}\right)^{4}\left(m_{K} / m_{\pi}\right)^{2}\left(M_{W} / M_{H^{ \pm}}\right)^{4}$. Additionally, NP contributions to Kaon mixing appear only at the loop-level. The constraints from Kaon physics are satisfied for values of the Yukawa couplings smaller than about 0.1 and charged Higgs masses larger than $250 \mathrm{GeV}$, as required to describe the $W+2$ jets data within this model.

For the case of $U_{u, L}^{i j}=V_{\mathrm{CKM}}^{* j i}$, the flavor violating coupling of the c- and u-quarks to $\phi_{2}^{0}$ leads to a generically large tree-level contribution to the $D-\bar{D}$ mixing when the scalar and pseudoscalar masses are non-degenerate. In all generality, we can define $y_{c u}$ the coupling $\phi_{2}^{0} \bar{u}_{L, 2} u_{1}$. In figure 2, we show the constraint on $y_{c u}$ as a function of the mass splitting $M_{A^{0}}-M_{H^{0}}$. The fit is performed using the experimental values given by HFAG [31]. As figure 2 suggests, even a few GeV splitting between $M_{H^{0}}$ and $M_{A^{0}}$ requires that $y_{c u} \lesssim 10^{-3}$. This bound can be read as a bound on the coupling $y_{u, 2}$ for the scenario with $U_{u, L}^{i j}=V_{\mathrm{CKM}}^{* j i}$ $\left(y_{c u}=y_{u, 2} V_{\mathrm{CKM}}^{* 21}\right)$. We can conclude that for this scenario to be viable we need $H^{0}$ and $A^{0}$ to be almost degenerate in mass.

A comparison of these two flavor scenarios suggests that the flavor constraints on the $U_{u, L}^{i j}=\delta^{i j}$ scenario are weaker and a substantial splitting in the Higgs masses is allowed. With sizable splittings between the Higgs masses $M_{H^{0}}$ and $M_{A^{0}}$, the electroweak precision tests are consistent with a lighter Standard Model-like Higgs, as is shown in figure 2. For the $U_{u, L}^{i j}=V_{\mathrm{CKM}}^{* j i}$ scenario, instead, the flavor constraints typically require a small splitting between the $H^{0}$ and $A^{0}$ Higgs masses and hence a heavier Standard Model-like Higgs boson is preferred in this case.

\section{$4 W^{+} H^{0} / W^{+} A^{0}$ production and $\mathrm{W}+$ jets excess}

The signal process of interest to us is

$$
p \bar{p} \rightarrow H^{ \pm} \rightarrow W^{ \pm} H^{0} / W^{ \pm} A^{0} \rightarrow \ell^{ \pm} \nu j j,
$$

where the charged lepton comes from the $W$-boson decay. The underlying parton level process is shown in figure 3. This is the dominant source of $W^{ \pm} H^{0} / A^{0}$ production since the charged Higgs is produced resonantly. In addition there are t-channel processes that contribute to the total signal rate. Inverting the hierarchy between the neutral and charged Higgs boson, one could also consider the process

$$
p \bar{p} \rightarrow H^{0} / A^{0} \rightarrow W^{ \pm} H^{\mp} \rightarrow \ell^{ \pm} \nu j j .
$$




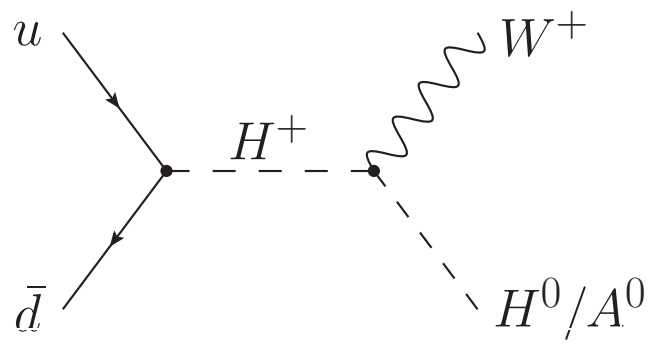

Figure 3. Dominant contribution to $W^{ \pm} H^{0} / A^{0}$ production at the parton level.

\begin{tabular}{|c|c|c|c|c|c|}
\hline$M_{H^{ \pm}}[\mathrm{GeV}], y_{u, 2}^{11}$ & $\Gamma_{H^{ \pm}}[\mathrm{GeV}]$ & $\operatorname{Br}\left(H^{ \pm} \rightarrow W^{ \pm} \varphi^{0}\right)$ & $\sigma\left(W^{ \pm} \varphi^{0}\right)$ & $\sigma\left(Z \varphi^{0}\right)$ & $\sigma\left(\gamma \varphi^{0}\right)$ \\
\hline $300,0.06$ & 4.45 & $97.4 \%$ & 2.04 & 0.12 & 0.11 \\
\hline $250,0.06$ & 0.587 & $84 \%$ & 3.74 & 0.12 & 0.11 \\
\hline
\end{tabular}

Table 1. Branching fractions and production cross sections for two benchmark points. The remaining parameters are $M_{H^{0}}=M_{A^{0}}=150 \mathrm{GeV}$. We display the total width of $H^{ \pm}$and the branching fraction into $W^{ \pm} \varphi^{0}$, where $\varphi^{0}=\left\{H^{0}, A^{0}\right\}$. The last three columns show the cross sections $\sigma\left(p \bar{p} \rightarrow X \varphi^{0}\right)$ in pb at the Tevatron. For the photon $p_{T}>20 \mathrm{GeV}$ is required.

This will have essentially a very similar collider signature and comparable effects in flavor and electroweak measurements.

In addition to the desired signal, our model also contributes to the $Z+j j$ and $\gamma+j j$ channels, through the processes

$$
\begin{aligned}
& p \bar{p} \rightarrow Z H^{0} / A^{0} \rightarrow Z j j, \quad p \bar{p} \rightarrow A^{0} \rightarrow Z H^{0} \rightarrow Z j j, \\
& p \bar{p} \rightarrow \gamma H^{0} / A^{0} \rightarrow \gamma j j .
\end{aligned}
$$

Since these processes are not mediated by on-shell resonances, they are suppressed compared to the $W^{ \pm} j j$ channel. The tree-level cross sections for these channels at the Tevatron are listed in table 1 .

The mass of the scalar $H^{0}$ is fixed to $150 \mathrm{GeV}$ in order to reproduce the excess in the dijet invariant mass spectrum. We calculate the cross sections for $W^{ \pm} H^{0} / A^{0}, Z H^{0} / A^{0}$ and $\gamma H^{0} / A^{0}$ at the Tevatron for two values of the charged Higgs mass, $M_{H^{ \pm}}=250 \mathrm{GeV}$ and $M_{H^{ \pm}}=300 \mathrm{GeV}$. Larger splittings between the neutral and charged Higgs states are disfavored by EWPTs. The remaining free parameter is the up quark Yukawa coupling $y_{u, 2}^{11}$, which is chosen such that the required amount of signal events is obtained. As discussed in the previous section, we shall assume that both $y_{u, 2}^{21}$ and $y_{u, 2}^{31}$ are smaller than $y_{u, 2}^{11}$ and hence they have no impact in the particle production at the Tevatron. We shall briefly discuss on the possible effects of a different coupling arrangement in the conclusions of this article.

The signal is generated using CalcHEP/CompHEP [32, 33] (and checked independently with MadGraph [34]), and then processed through Pythia [35] for parton showering and hadronization and through PGS for a detector simulation, for which the CDF parameter set is used. 


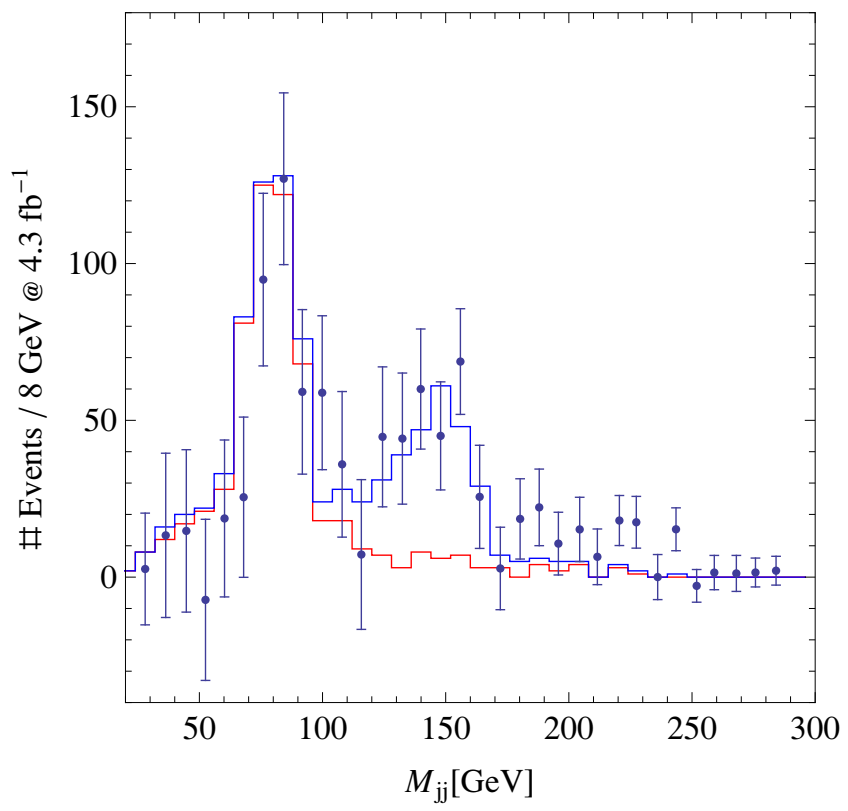

Figure 4. Dijet invariant mass spectrum for di-boson background only (red) and signal plus background (blue). The parameters chosen for the simulation are $M_{H^{ \pm}}=300 \mathrm{GeV}, M_{H}^{0}=M_{A}^{0}=$ $150 \mathrm{GeV}$ and $y_{u, 2}^{11}=0.06$. The data points are taken from figure 1 in [1].

We implement the cuts of the CDF analysis: for the leptons, we require $p_{T}>20 \mathrm{GeV}$ and $|\eta|<1$. In addition, a missing energy greater than $30 \mathrm{GeV}$ is required, and the transverse mass of the lepton $+E_{T}$ system is required to be larger than $30 \mathrm{GeV}$. Jets are reconstructed with a cone size $R=0.4$ and required to have $p_{T}>30 \mathrm{GeV},|\eta|<2.4$ and $\Delta \eta_{j j}<2.5$. Further the transverse momentum of the dijet system is required to have $\left|p_{T j_{1}}+p_{T j_{2}}\right|>40 \mathrm{GeV}$. Events with more than two jets are rejected, as well as events with an additional lepton with $p_{T}>20 \mathrm{GeV}$. We also reject events where the lepton is within a $\Delta R \leq 0.52$ cone around either of the two jets.

To correct the jet energy scale, we also simulate the $W+2$ jets signal from $W^{+} W^{-}$and $W^{ \pm} Z$ production. In order to compensate the mis-reconstruction of the $W$-boson peak in PGS, we multiply the jet energy by 1.1 , such that the mass of the hadronically decaying $W$-boson is at $80 \mathrm{GeV}$. The same scaling is applied to the signal.

The result of the simulation for di-boson background only and for signal plus di-boson background is shown in figure 4 . To match the height of the W-boson peak to data we multiply the tree-level cross sections for $W W$ and $W Z$ production by a K-factor of 1.4. For $M_{H^{ \pm}}=300 \mathrm{GeV}, 17 \%$ of the events with $W \rightarrow \ell \nu_{\ell},(\ell=e, \mu)$ pass the cuts, while for $M_{H^{ \pm}}=250 \mathrm{GeV}$ this number drops to $8.7 \%$. A good fit to the dijet invariant mass spectrum is found using 300 signal events, corresponding to a coupling $y_{u, 2}^{11}=0.06$ for both $M_{H^{ \pm}}=300 \mathrm{GeV}$ and $M_{H^{ \pm}}=250 \mathrm{GeV}$.

In ref. [10] a different set of cuts was suggested for the CDF analysis that may improve the signal to background ratio by a factor of two. In particular, to be more sensitive to the $W+$ resonance structure of the event, they suggest a cut on the sum of lepton and missing 
transverse momentum, $\left|p_{T \ell}+p_{T \nu_{\ell}}\right|>60 \mathrm{GeV}$, and require the angular separation between the jets to be $\Delta \phi\left(j_{1}, j_{2}\right)>1.75$. For our model with $M_{H^{ \pm}}=300 \mathrm{GeV}$, these cuts reduce the signal by $32 \%$. Comparing with the results of ref. [10] we expect a similar increase of the signal to background ratio in our case.

Let us finally comment on the effects of splitting the masses of the neutral Higgs bosons $H^{0}$ and $A^{0}$. Small mass differences have only a minor influence on the signal rate. For large mass differences, $\delta m_{H^{0} A^{0}} \geq 50 \mathrm{GeV}$, as required for a significant improvement of the fit to precision electroweak data for moderate values of the SM Higgs boson mass, the dominant signal rate is associated with the lightest of these Higgs bosons, while the other one leads to a small or no signal contribution. This is, for instance, the case for the values $M_{H^{0}} \simeq 150 \mathrm{GeV}, M_{A^{0}} \simeq 210 \mathrm{GeV}$, and $M_{H^{ \pm}} \simeq 300 \mathrm{GeV}$, used in figure 1. Although the charged Higgs may decay to on-shell $W$ and $A^{0}$ bosons, the phase space suppression is strong enough to lead to only a very minor increase of the signal in the $M_{j j} \simeq 210 \mathrm{GeV}$ region. This region can however be populated if also the mass of the charged Higgs is increased (see appendix).

\section{Prospects at Tevatron and LHC}

At hadron colliders, the $150 \mathrm{GeV}$ dijet resonance can be produced also in association with a $Z$ boson or with a photon. In our model, the main production of the $W+$ jets signal is through resonant charged Higgs production. Hence, the $\gamma+$ jets and $Z+$ jets signals at the Tevatron, which are only produced in the t-channel, are suppressed by more than an order of magnitude with respect to the $W+2$ jets one (c.f. table 1). Assuming a similar signal acceptance, and taking into account the small branching fraction of the $Z$ boson to lepton pairs, we do not expect the Tevatron to see any relevant excess in the $(Z \rightarrow \ell \ell) j j$ channel [36] (p. 24).

In the photon plus dijet channel at the Tevatron, we expect up to 40 events for a photon with $p_{T}>20 \mathrm{GeV}$. This channel is dominated by QCD dijet production with an additional photon. A search in this channel has been performed with very mild cuts on the jet energies [37], and our model is consistent with these observations. A more refined analysis with optimized cuts could provide important constraints on this and other models for the $W+$ jets CDF excess.

At the LHC, it is possible to search for all of the above signals. In addition, the larger center of mass energy allows for sizable production cross sections also for $Z H^{ \pm}, W^{ \pm} H^{\mp}$ and for pair production of scalars. The cross sections at the LHC with $\sqrt{s}=7 \mathrm{TeV}$ are given in table 2 .

One promising signal comes from Drell-Yan production of $H^{+} H^{-}$pairs, that gives rise to $W^{+} W^{-}+4$ jets signals. The main background for this process is $t \bar{t}$ production with additional jets. This background can be reduced by explicitly asking for four hard jets, and demanding that the invariant mass of pairs of jets is close to the mass of the resonance. In addition, a veto on $\mathrm{b}$ tagged jets may be useful to discriminate between the signal and background. 


\begin{tabular}{|c|c|c|c|c|c|c|}
\hline$M_{H^{ \pm}}[\mathrm{GeV}], y_{u, 2}^{11}$ & $\sigma\left(W^{ \pm} \varphi^{0}\right)$ & $\sigma\left(Z \varphi^{0}\right)$ & $\sigma\left(\gamma \varphi^{0}\right)$ & $\sigma\left(W^{ \pm} H^{\mp}\right)$ & $\sigma\left(Z H^{ \pm}\right)$ & $\sigma\left(H^{+} H^{-}\right)$ \\
\hline $300,0.06$ & 7.72 & 0.35 & 0.31 & 0.026 & 0.011 & 0.0010 \\
\hline $250,0.06$ & 13.7 & 0.35 & 0.31 & 0.034 & 0.016 & 0.0023 \\
\hline
\end{tabular}

Table 2. LHC production cross sections for two benchmark points. The remaining parameters are $M_{H^{0}}=M_{A^{0}}=150 \mathrm{GeV}$. Shown are the production cross sections (in pb) at LHC with $\sqrt{s}=7 \mathrm{TeV}$ for several promising channels. For the photon $p_{T}>20 \mathrm{GeV}$ is required.

\section{Conclusions}

We have presented a simple renormalizable model that can explain the CDF excess in $W+2$ jets that was recently observed by the CDF collaboration. Our model is in agreement with all constraints coming from electroweak precision measurements, and depending on the precise values of the new charged and neutral Higgs boson masses, allows a broad range of the Standard Model Higgs mass from $100 \mathrm{GeV}$ to several hundreds of GeV.

The model predicts no significant signal in the $Z+2$ jets and in the $W b \bar{b}$ channel, in agreement with current experimental constraints on these channels. At the LHC, the model can be searched for in dijets produced in association with an electroweak gauge boson. In addition, we also expect a sizable production cross section for $W W+$ jets and $Z W+$ jets at the LHC. These signals might be easier to separate from the notoriously large QCD background.

Given the steadily growing number of models that attempt to explain the CDF excess, it might be worth considering possibilities to discriminate between different models. In principle angular observables should be suitable for this task, since angular distributions typically depend on the spin of intermediate resonances and on the production mode.

More concretely, we expect t-channel $Z^{\prime}$ models to give a different $p_{T}$ spectrum (softer and more forward) than in the s-channel production considered in this work. Moreover, no resonant feature on the total invariant mass of the $W+j j$ system is expected in those models. Considering there is only one leptonic $W$, we can fully reconstruct the event and study the spin of various resonances. In our particular case, the spin of $H^{+}$may be studied using the $W H^{0}$ angular distribution in the rest frame of $H^{+}$. As stressed before, the relative ratio of $W+j j$ rate to the $Z+j j$ (or $\gamma+\mathrm{jj}$ ) is a very good diagnostic that may distinguish different models.

We finally want to mention that, had we assumed $y_{u, 2}^{31}$ to be the most significant coupling, a relevant signal could still be obtained for large values of this coupling, $y_{u, 2}^{31} \simeq 1.5$, via the $u \bar{b} \rightarrow H^{+} \rightarrow H^{0} / A^{0}+j j$ and $g u \rightarrow t H^{0} / A^{0}$ channels. We studied this possibility and found that the rate in the former channel is a factor four larger than the latter and therefore the signal characteristics are very similar to the one studied in this article. Similarly to what happens in the model of ref. [13], such a coupling arrangement leads also to an increase of the top-quark forward-backward asymmetry, but is subject to constraints coming from flavor physics and top-quark decays [38]. Further discussion on these possibilities will be studied elsewhere. 


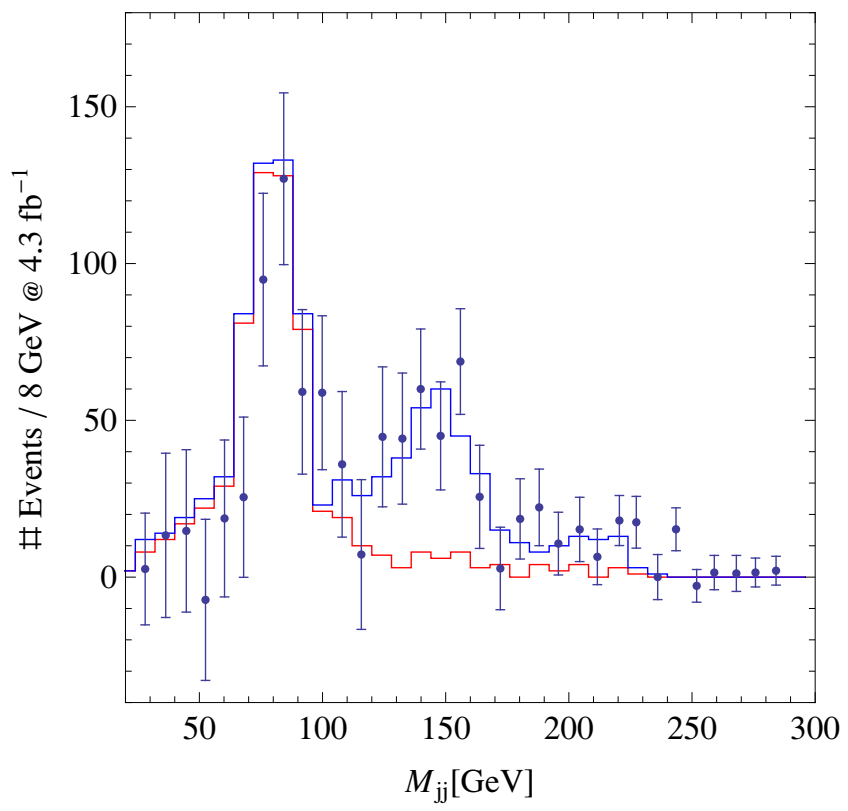

Figure 5. Dijet invariant mass spectrum for di-boson background only (red) and signal plus background (blue). The parameters chosen for the simulation are $M_{H^{ \pm}}=330 \mathrm{GeV}, M_{A^{0}}=210 \mathrm{GeV}$ and $M_{H^{0}}=150 \mathrm{GeV}$.

\section{Acknowledgments}

We thank W. Altmannshofer and R. Culbertson for useful discussions. Work at ANL is supported in part by the U.S. Department of Energy (DOE), Div. of HEP, Contract DE-AC02-06CH11357. Fermilab is operated by the Fermi Research Alliance, LLC under Contract No DE-AC02-07CH11359 with the U.S. Department of Energy. P.S. is partially supported by the UIC DOE HEP Contract DE-FG02-84ER40173. L.-T.W. is supported by the DOE Early Career Award under grant DE-SC0003930. A.M. is supported by the U.S. Department of Energy under Contract No. DE-FG02-94ER40840.

\section{A Alternative spectrum}

The mass spectrum of the model is strongly constrained by precision constraints. Mass splittings between the scalars larger than the ones considered above are very hard to bring in agreement with these constraints. However, one could also view this model as an effective theory valid up to around the $\mathrm{TeV}$ scale, where additional new physics is expected to solve the hierarchy problem. In this case, additional new physics contributions to the electroweak $\mathrm{S}$ and $\mathrm{T}$ parameters will in general be present. These may partially compensate the contributions from the Higgs doublet, thus making larger mass splittings phenomenologically viable.

Although the data in the invariant mass bins between $180 \mathrm{GeV}$ and $240 \mathrm{GeV}$ lie systematically above the expected background, this excess is not statistically significant, and in addition, is subject to possibly large NLO uncertainties [36] (p. 33). Consequently, 
no preference for a model leading to such an excess can be claimed, on the other hand, the data is not in conflict with this possibility. As an example of such a model, in figure 5 we show the dijet invariant mass distribution for $M_{H^{ \pm}}=330 \mathrm{GeV}, M_{A}=210 \mathrm{GeV}$ and $M_{H^{0}}=150 \mathrm{GeV}$. In this case our model fits well the data up to invariant masses of $230 \mathrm{GeV}$, while only a small increase of the coupling $y_{u, 2}$ is required.

Open Access. This article is distributed under the terms of the Creative Commons Attribution Noncommercial License which permits any noncommercial use, distribution, and reproduction in any medium, provided the original author(s) and source are credited.

\section{References}

[1] CDF collaboration, T. Aaltonen et al., Invariant Mass Distribution of Jet Pairs Produced in Association with a $W$ boson in pp Collisions at $\sqrt{s}=1.96 \mathrm{TeV}$, Phys. Rev. Lett. 106 (2011) 171801 [arXiv: 1104.0699] [SPIRES].

[2] M.R. Buckley, D. Hooper, J. Kopp and E. Neil, Light Z' Bosons at the Tevatron, Phys. Rev. D 83 (2011) 115013 [arXiv:1103.6035] [SPIRES].

[3] F. Yu, A Z' Model for the CDF Dijet Anomaly, Phys. Rev. D 83 (2011) 094028 [arXiv: 1104.0243] [SPIRES].

[4] K. Cheung and J. Song, Baryonic Z' Explanation for the CDF Wjj Excess, Phys. Rev. Lett. 106 (2011) 211803 [arXiv:1104.1375] [SPIRES].

[5] L.A. Anchordoqui, H. Goldberg, X. Huang, D. Lüst and T.R. Taylor, Stringy origin of Tevatron Wjj anomaly, Phys. Lett. B 701 (2011) 224 [arXiv:1104.2302] [SPIRES].

[6] M. Buckley, P. Fileviez Perez, D. Hooper and E. Neil, Dark Forces At The Tevatron, arXiv:1104.3145 [SPIRES].

[7] P. Ko, Y. Omura and C. Yu, Dijet resonance from leptophobic Z' and light baryonic cold dark matter, arXiv:1104.4066 [SPIRES].

[8] P.J. Fox, J. Liu, D. Tucker-Smith and N. Weiner, An Effective Z', arXiv:1104.4127 [SPIRES].

[9] D.-W. Jung, P. Ko and J.S. Lee, A Possible Common Origin of the Top Forward-backward Asymmetry and the CDF Dijet Resonance, arXiv:1104.4443 [SPIRES].

[10] E.J. Eichten, K. Lane and A. Martin, Technicolor at the Tevatron, arXiv:1104.0976 [SPIRES].

[11] C. Kilic and S. Thomas, Signatures of Resonant Super-Partner Production with Charged-Current Decays, arXiv:1104.1002 [SPIRES].

[12] J.L. Christiansen et al., $O(100 \mathrm{GeV})$ Deci-weak $W^{\prime} / Z^{\prime}$ at Tevatron and LHC, Phys. Rev. D 83 (2011) 117701 [arXiv:1104.1161] [SPIRES].

[13] A.E. Nelson, T. Okui and T.S. Roy, A unified, flavor symmetric explanation for the $t \bar{t}$ asymmetry and Wjj excess at CDF, arXiv:1104.2030 [SPIRES].

[14] R. Sato, S. Shirai and K. Yonekura, A Possible Interpretation of CDF Dijet Mass Anomaly and its Realization in Supersymmetry, Phys. Lett. B 700 (2011) 122 [arXiv:1104.2014] [SPIRES]. 
[15] X.-P. Wang, Y.-K. Wang, B. Xiao, J. Xu and S.-h. Zhu, New Color-Octet Vector Boson Revisit, Phys. Rev. D 83 (2011) 115010 [arXiv:1104.1917] [SPIRES].

[16] X.-G. He and B.-Q. Ma, The CDF dijet excess from intrinsic quarks, arXiv:1104.1894 [SPIRES].

[17] B.A. Dobrescu and G.Z. Krnjaic, Weak-triplet, color-octet scalars and the CDF dijet excess, arXiv:1104.2893 [SPIRES].

[18] Z. Sullivan and A. Menon, Standard model explanation of a CDF dijet excess in Wjj, Phys. Rev. D 83 (2011) 091504 [arXiv:1104.3790] [SPIRES].

[19] T. Plehn and M. Takeuchi, W+Jets at CDF: Evidence for Top Quarks, arXiv:1104.4087 [SPIRES].

[20] E. Ma, Verifiable radiative seesaw mechanism of neutrino mass and dark matter, Phys. Rev. D 73 (2006) 077301 [hep-ph/0601225] [SPIRES].

[21] L. Lopez Honorez, E. Nezri, J.F. Oliver and M.H.G. Tytgat, The inert doublet model: An archetype for dark matter, JCAP 02 (2007) 028 [hep-ph/0612275] [SPIRES].

[22] H.K. Dreiner, M. Hanussek and S. Grab, Bounds on R-parity Violating Couplings at the Grand Unification Scale from Neutrino Masses, Phys. Rev. D 82 (2010) 055027 [arXiv: 1005.3309] [SPIRES].

[23] H. Leutwyler, How about $m$ (u) =0?, Nucl. Phys. B 337 (1990) 108 [SPIRES].

[24] MILC collaboration, C. Aubin et al., Light pseudoscalar decay constants, quark masses and low energy constants from three-flavor lattice QCD, Phys. Rev. D 70 (2004) 114501 [hep-lat/0407028] [SPIRES].

[25] Particle Data Group collaboration, K. Nakamura et al., Review of particle physics, J. Phys. G 37 (2010) 075021 [SPIRES].

[26] H. Davoudiasl and A. Soni, Strong CP, Up-Quark Mass and the Randall-Sundrum Microscope, Phys. Rev. D 76 (2007) 095015 [arXiv:0705.0151] [SPIRES].

[27] http://gfitter.desy.de/Figures/GOblique/S_vs_T_logo_full.gif.

[28] P.H. Chankowski, M. Krawczyk and J. Zochowski, Implications of the precision data for very light Higgs boson scenario in 2HDM(II), Eur. Phys. J. C 11 (1999) 661 [hep-ph/9905436] [SPIRES].

[29] P.H. Chankowski et al., Do precision electroweak constraints guarantee $e^{+} e^{-}$collider discovery of at least one Higgs boson of a two Higgs doublet model?, Phys. Lett. B 496 (2000) 195 [hep-ph/0009271] [SPIRES].

[30] https://twiki.cern.ch/twiki/bin/view/CMSPublic/PhysicsResultsHIGStandardModelProjections.

[31] http://www.slac.stanford.edu/xorg/hfag/charm/CHARM10/results_mix+cpv.html.

[32] A. Pukhov, Calchep 2.3: MSSM, structure functions, event generation, 1 and generation of matrix elements for other packages, hep-ph/0412191 [SPIRES].

[33] CompHEP collaboration, E. Boos et al., CompHEP 4.4: Automatic computations from Lagrangians to events, Nucl. Instrum. Meth. A 534 (2004) 250 [hep-ph/0403113] [SPIRES].

[34] J. Alwall et al., MadGraph/MadEvent v4: The New Web Generation, JHEP 09 (2007) 028 [arXiv:0706.2334] [SPIRES]. 
[35] T. Sjöstrand, S. Mrenna and P.Z. Skands, PYTHIA 6.4 Physics and Manual, JHEP 05 (2006) 026 [hep-ph/0603175] [SPIRES].

[36] http://theory.fnal.gov/jetp/talks/Viviana.pdf.

[37] CDF collaboration, Search for Anomalous Production of Photon + Jets + Missing Transverse Energy Events in ppbar collisions at $\sqrt{s}=1.96 \mathrm{TeV}$, CDF Conference Note 10355 (2010).

[38] G. Zhu, B physics constraints on a flavor symmetric scalar model to account for the ttbar asymmetry and Wjj excess at CDF, arXiv:1104.3227 [SPIRES]. 